\title{
Efeito do método Pilates em fatores de risco para doenças cardiometabólicas: uma revisão sistemática
}

\author{
Effect of Pilates exercises in risk factors for cardiometabolic diseases: \\ a systematic review
}

\author{
Silvana Junges, ${ }^{1}$ Camila Bittencourt Jacondino, ${ }^{2}$ Maria Gabriela Valle Gottlieb ${ }^{3} \bowtie$ \\ ${ }^{1}$ Educadora Física, Mestre em Ciências da Saúde e Doutoranda do Programa de Pós-Graduação em Gerontologia Biomédica do Instituto \\ de Geriatria e Gerontologia da Pontifícia Universidade Católica do Rio Grande do Sul (PUCRS). Porto Alegre, RS. \\ 2 Enfermeira, Mestre em Gerontologia Biomédica e Doutoranda do Programa de Pós-Graduação em Gerontologia Biomédica do Instituto \\ de Geriatria e Gerontologia da PUCRS. Porto Alegre, RS. \\ ${ }^{3}$ Bióloga, Mestre e Doutora em Ciências da Saúde, Pós-Doutora em Gerontologia Biomédica. Docente do Programa de Pós-Graduação \\ em Gerontologia Biomédica do Instituto de Geriatria e Gerontologia da PUCRS. Porto Alegre, RS.
}

Este estudo teve apoio do Programa Nacional de Pós-Doutorado da Coordenação de Aperfeiçoamento de Pessoal de Nível Superior (PNDP/CAPES).

\section{RESUMO}

Objetivos: realizar uma revisão sistemática da literatura sobre o efeito do método Pilates em fatores de risco para doenças cardiometabólicas. Métodos: A busca foi realizada nas bases de dados LILACS, SciELO, PubMed e Scopus, nos idiomas inglês e português. Os termos de busca utilizados foram exercícios de Pilates com as seguintes palavras chaves e seus correlatos em inglês: sedentarismo, obesidade, índice de massa corporal, composição corporal, pressão arterial, diabetes mellitus e dislipidemia. Os critérios de inclusão foram estudos de intervenção com Pilates, artigos originais e disponíveis na íntegra em inglês e português, população alvo portadora de pelo menos um fator de risco cardiometabólico e sedentários. Estudos que não preencheram esses critérios foram excluídos. O período de captura dos artigos foi de 2004 a 2014 e a elaboração do artigo ocorreu entre julho de 2014 e janeiro de 2015.

Resultados: foram encontrados 93 artigos, sendo que apenas sete atenderam aos critérios de seleção. Destes, três estudos (42,86\%) investigaram o efeito do Pilates em fatores de risco cardiometabólicos clássicos: triglicerídeos, glicose e lipoproteína de alta densidade ligada ao colesterol, porém não foram encontrados resultados benéficos nesses parâmetros no grupo intervenção com Pilates. Por outro lado, dos sete estudos incluídos, seis deles $(85,71 \%)$ verificaram reduções estatisticamente significativas em algum dos seguintes fatores: pressão arterial sistólica e diastólica, índice de massa corporal, percentual de gordura corporal, massa gorda e relação cintura-quadril entre os grupos intervenção com Pilates e controle $(\mathrm{P}<0,05)$.

Conclusões: O Pilates está se consolidando como um exercício eficaz na redução de porcentagem de gordura corporal, massa gorda, relação cintura/quadril e da pressão arterial em indivíduos de diferentes faixas etárias. Esses benefícios, principalmente da redução da relação cintura/ quadril e pressão arterial têm um impacto clinicamente relevante na morbimortalidade por doenças cardiovasculares e cerebrovasculares. Contudo, mais estudos randomizados e controlados necessitam ser conduzidos nessa área.

DESCRITORES: pilates; exercício físico; modalidades de fisioterapia; doenças cardiovasculares; diabetes mellitus; obesidade.

\section{ABSTRACT}

Aims: To perform a systematic review of the literature on the effect of Pilates on risk factors for cardiometabolic diseases.

Methods: The search was conducted in the LILACS, SciELO, PubMed, and Scopus databases both in English and in Portuguese. The search term used was Pilates exercise combined with the following key words and their equivalents in Portuguese: sedentary lifestyle, obesity, body mass index, body composition, blood pressure, diabetes mellitus, and dyslipidemia. The inclusion criteria consisted of intervention studies on Pilates, original articles in English and Portuguese available in their full-length versions, and target population with at least one cardiometabolic risk factor and with a sedentary lifestyle. Studies that failed to meet these criteria were excluded. The articles were collected from 2004 to 2014, and this review was written between July 2014 and January 2015.

Results: Ninety-three articles were selected, but only seven met the selection criteria. Of these, three studies (42.86\%) investigated the effect of Pilates on classic cardiometabolic risk factors: triglycerides, glucose, and high-density lipoprotein cholesterol, but no beneficial results were obtained for these parameters in the intervention group. On the other hand, six (85.71\%) out of the seven studies, revealed statistically significant reductions in some of the following factors: systolic and diastolic blood pressure, body mass index, body fat percentage, fat mass, and waist-to-hip ratio between the intervention and control groups $(\mathrm{P}<0.05)$.

Conclusions: Pilates has proven effective in reducing body fat percentage, fat mass, waist-to-hip ratio, and systemic blood pressure in different-aged individuals. These benefits, mainly the reduction of waist-to-hip ratio and of systemic blood pressure, have a clinically relevant impact on morbidity and mortality from cardiovascular and cerebrovascular diseases. However, more randomized controlled trials need to be conducted in this area.

KEY WORDS: pilates; exercise; physical therapy modalities; cardiovascular diseases; diabetes mellitus; obesity. 


\section{INTRODUÇÃO}

Atualmente, a discussão sobre o desencadeamento de doenças crônicas não transmissíveis, como as cardiovasculares, tem se concentrado em torno de fatores genéticos, metabólicos e estilo de vida, principalmente relacionadas à atividade física e dieta. Tal discussão e estudos científicos são de grande relevância em nível de saúde pública, uma vez que as doenças cardiovasculares (DCV) representam a principal causa de morbimortalidade em países desenvolvidos e em desenvolvimento, como é o caso do Brasil [1]. Neste contexto, são considerados como principais fatores de risco cardiovascular: 1) história familiar de doença arterial coronariana prematura (familiar de primeiro grau do sexo masculino que apresentou a doença com menos 55 anos e do sexo feminino com menos de 65 anos); 2) ser homem com idade acima de 45 anos e mulher com idade acima de 55 anos; 3) tabagismo; 4) hipercolesterolemia: lipoproteína de baixa densidade ligada ao colesterol (LDL-c) elevada; 5) hipertensão arterial sistêmica (HAS): pressão diastólica acima de $90 \mathrm{~mm} \mathrm{Hg}$ e sistólica acima de $140 \mathrm{~mm} \mathrm{Hg}$; 6) diabetes mellitus (DM) tipo $2 ; 7$ ) obesidade: índice de massa corporal (IMC) de $30 \mathrm{~kg} / \mathrm{m}^{2}$ ou mais; 8) gordura abdominal (medida pela circunferência da cintura, onde os pontos de corte adotados são: mulheres $>80,0 \mathrm{~cm}$ e homens $>94,0 \mathrm{~cm}$ ); 9) sedentarismo; 10) dieta pobre em frutas e vegetais; 11) estresse psicossocial. Adicionalmente, os fatores de risco cardiometabólicos são aqueles implicados no desenvolvimento de DM tipo 2 e DCV, devido a um conjunto de fatores de risco modificáveis [1-3]. Nesse sentido, diversos estudos têm demonstrado que o sedentarismo é um dos principais fatores de risco que podem contribuir para o desencadeamento de distúrbios metabólicos e DCV [4-7].

Por outro lado, é preciso destacar que a prática de exercício físico regular, como por exemplo, a aplicação do método Pilates, tem potencial de prevenir fatores de risco cardiometabólicos, uma vez que concilia exercício postural e de concentração, com foco na musculatura das costas e abdominal $[1,8,9]$. Os estudos têm mostrado que o Pilates é eficaz em reduzir a adiposidade intra-abdominal, que é metabolicamente ativa, produzindo inflamação crônica de baixo grau, aumento do fluxo de ácidos graxos livres e aumento nas taxas de LDL-c no plasma [10,11]. Isso pode acarretar o comprometimento da função de órgãos como fígado, pâncreas e músculo esquelético, devido ao acúmulo ectópico de lipídeos e à formação de metabólitos tóxicos, causando danos intracelulares e alterações nas enzimas mitocondriais, levando ao acúmulo intracelular de glicose. Esse acúmulo de glicose pode desencadear resistência à insulina, a qual está associada à disfunção endotelial e ao subsequente desencadeamento de DCV [12-14].

$\mathrm{O}$ método Pilates foi fundado por Joseph Pilates durante a década de 1920. Sua ênfase está sobre o controle do corpo, posição, movimento e mente, fazendo com que se denominasse originalmente como Arte do Controle ou "Contrologia" [15]. Os princípios básicos do método Pilates incluem centralização, concentração, controle, precisão, fluidez e respiração [16]. Os exercícios podem ser executados no solo sem uso de aparelhos (Mat Pilates), ou envolver o uso de equipamentos especializados que oferecem resistência ajustável. O método Pilates pode ser indicado para qualquer indivíduo, de diferentes faixas etárias, sendo mais frequentemente prescrito para pessoas com dor lombar crônica, para correção postural e melhora do equilíbrio dinâmico, da flexibilidade, da força e da resistência, para o aumento da massa muscular e redução de massa gorda, para reabilitação e para a melhoria da qualidade de vida $[17,18]$.

Atualmente, o Pilates vem se consolidando como um exercício físico com elevado número de adeptos em diversos países. Nos Estados Unidos, por exemplo, é uma das principais práticas físicas, com aproximadamente 10,5 milhões de praticantes, não apenas para correção postural, como também para prevenção dos fatores de risco para DCV e doenças metabólicas [9-11,19]. Junges et al. [9] verificaram a eficácia de um programa de Pilates por um período de 30 semanas (frequência de duas vezes por semana) na redução da porcentagem de gordura corporal, da relação cintura/quadril e do grau de cifose em mulheres pós-menopáusicas. Os resultados desse e de outros estudos sugerem que o gasto calórico e a correção postural promovidas pelo Pilates podem resultar na melhoria da distribuição do tecido adiposo, através da maior mobilização da gordura depositada na região abdominal $[9,15,16,20]$. Levando em conta essa ampliação das indicações do método, o presente estudo teve como propósito realizar uma revisão sistemática da literatura sobre o efeito do Pilates em fatores de risco para doenças cardiometabólicas.

\section{MÉTODOS}

Foi realizada uma busca de artigos científicos publicados no período de 2004 a 2014, por meio das bases de dados LILACS, SciELO, PubMed e Scopus. Utilizaram-se os descritores Exercícios de Pilates/Pilates Exercise, adicionando aos seguintes: sedentarismo/sedentary lifestyle, obesidade/obesity, 
índice de massa corporal/body mass index, composição corporal/body composition, pressão arterial/blood pressure, diabetes mellitus/diabetes mellitus e dislipidemia/dyslipidemia.

A revisão sistemática tem o início com a definição adequada da pergunta norteadora, existindo quatro componentes para a formulação de uma questão pesquisa, através da sigla $\mathrm{PICO}$, em que $\mathrm{P}=$ participante, $\mathrm{I}=$ intervenção, $\mathrm{C}=$ controle e $\mathrm{O}=$ desfecho [21]. A revisão sistemática do presente estudo foi norteada pela seguinte questão de pesquisa: Qual é o efeito do método Pilates em fatores de risco para as doenças cardiometabólicas?

Tendo como base esta informação, elegemos os seguintes critérios de inclusão: $\mathbf{P}=$ população do estudo portadora de pelo menos algum fator de risco cardiometabólico como sedentarismo, sobrepeso/ obesidade, diabetes mellitus, dislipidemia e hipertensão arterial; $\mathbf{I}=$ intervenção através do método Pilates; $\mathbf{C}=$ grupo que não recebeu intervenção; $\mathbf{O}=$ efeito significativo nas variáveis antropométricas (composição corporal, índice de massa corporal circunferência de cintura, relação cintura e quadril), glicemia de jejum, perfil lipídico e pressão arterial. Além disso, os artigos selecionados deveriam ser artigos originais, disponíveis na íntegra e redigidos nos idiomas inglês ou português.

\section{RESULTADOS DA SELEÇÃO}

A busca dos descritores na literatura resultou em um total de 93 artigos. Destes, 86 foram excluídos: 32 eram duplicatas, seis eram estudos de revisão, dois eram estudos com delineamento observacional, 35 estudos focavam outro tipo de desfecho que não fosse doença cardiometabólica e 11 estudavam outro tipo de exercício físico que não o Pilates, sendo selecionados somente sete artigos (Figura 1).

A presente revisão sistemática foi conduzida pela questão norteadora baseada na eficácia do método Pilates em fatores de risco para doenças cardiometabólicos. A literatura é escassa quanto a esse tema e a maioria dos estudos com Pilates investiga principalmente os seus efeitos na composição corporal e porcentagem

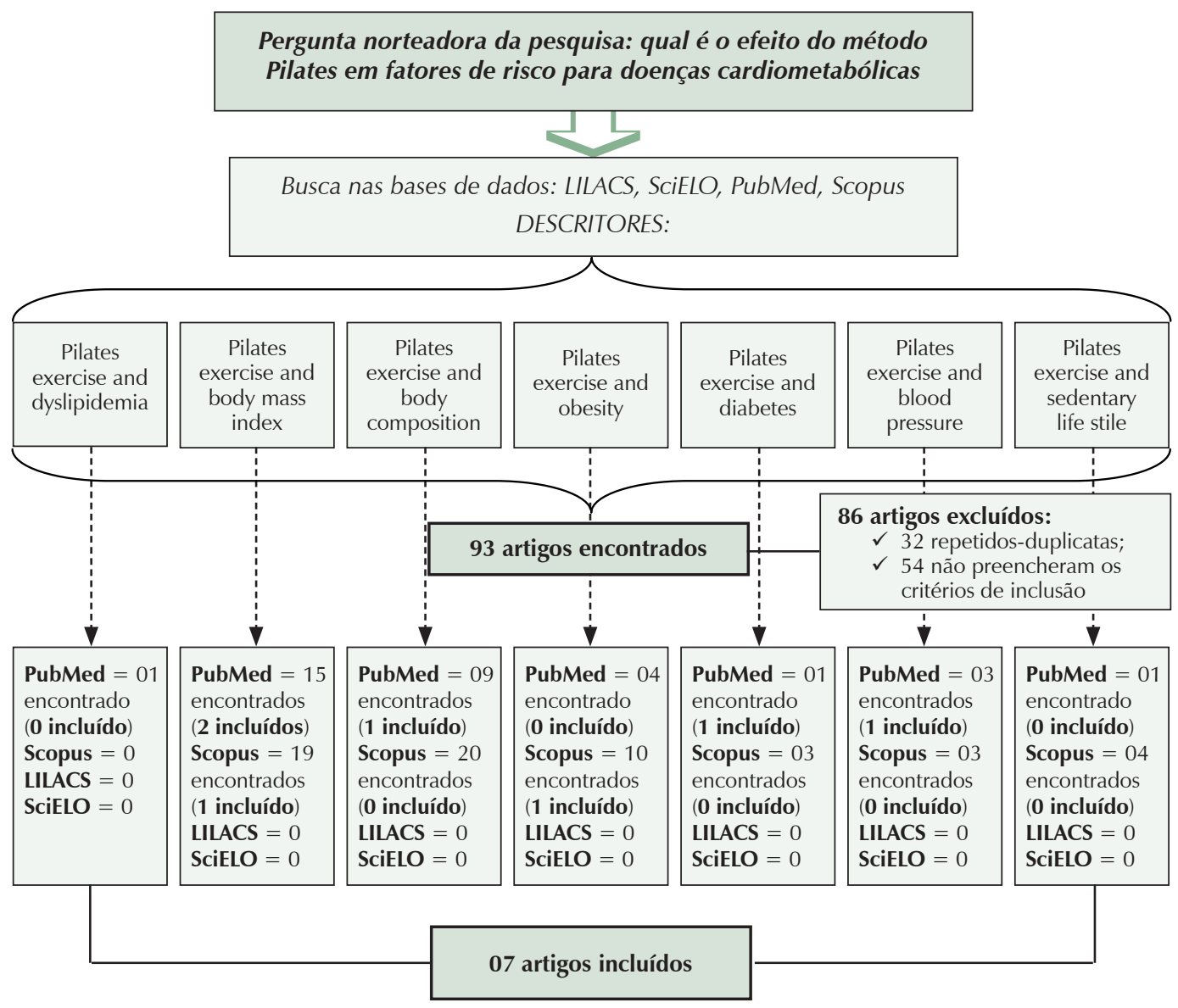

Figura 1. Etapas da revisão sistemática: busca, seleção e inclusão de estudos sobre o efeito do método Pilates em fatores de risco cardiometabólicos. 
de gordura corporal, bem como na dor lombar crônica e flexibilidade [22-25]. Além disso, o método Pilates é uma modalidade de exercício físico relativamente recente, e talvez por isso a dificuldade de encontrar e de incluir estudos com a questão norteadora proposta.

\section{CONTEÚDO DA REVISÃO}

Os sete estudos incluídos foram compostos majoritariamente por uma amostra adulta feminina e sedentária; apenas um estudo foi realizado com adolescentes e dois com idosos. Em relação à frequência, duração do exercício e da intervenção, constatou-se que um estudo utilizou quatro dias semanais de intervenção, um utilizou dois dias semanais e os restantes utilizaram três vezes por semana. Já em relação à duração das sessões, cinco estudos realizaram durante uma hora e os outros dois durante 45 minutos. Quanto ao período de duração da intervenção, três estudos realizaram durante oito semanas e os demais por 10, 12 e 16 semanas. Todos os estudos utilizaram o método Mat Pilates [26-32] (Tabela 1).

Tabela 1. Principais achados de estudos que investigaram o efeito do método Pilates em fatores de risco cardiometabólicos no período de 2004 a 2014. Todos os programas de intervenção utilizaram o Mat Pilates.

\begin{tabular}{|c|c|c|c|c|}
\hline $\begin{array}{l}\text { Autores } \\
\text { Ano } \\
\text { País }\end{array}$ & $\begin{array}{c}\text { População } \\
\text { Amostra } \\
\text { Idade dos participantes }\end{array}$ & $\begin{array}{l}\text { Duração da sessão } \\
\text { Frequência semanal } \\
\text { Duração da intervenção }\end{array}$ & $\begin{array}{c}\text { Variáveis } \\
\text { cardiometabólicas } \\
\text { avaliadas }\end{array}$ & Principais resultados \\
\hline $\begin{array}{l}\text { Fourie } M \text { et al. [26] } \\
2013 \\
\text { África do Sul }\end{array}$ & $\begin{array}{l}50 \text { idosas sedentárias } \\
25 \text { idosas randomizadas } \\
\text { para o Gl } \\
\text { Média de idade: } \\
\mathrm{GC}=65,32 \pm 5,01 \\
\mathrm{Gl}=66,12 \pm 4,77\end{array}$ & $\begin{array}{l}60 \text { minutos } \\
3 \text { dias por semana } \\
8 \text { semanas }\end{array}$ & $\begin{array}{l}\text { Gordura corporal } \\
\text { IMC }\end{array}$ & $\begin{array}{l}\text { O Gl apresentou diminuição } \\
\text { estatisticamente significativa da } \\
\text { porcentagem de gordura corporal } \\
(p=0,0016)\end{array}$ \\
\hline $\begin{array}{l}\text { Tunar } M \text { et al. [27] } \\
2012 \\
\text { Turquia }\end{array}$ & $\begin{array}{l}31 \text { pacientes sedentários } \\
\text { portadores de diabetes tipo } 1 \\
\text { com idades entre } 12-17 \text { anos. } \\
14 \text { pessoas foram submetidas a } \\
\text { sessões de Pilates } \\
\text { Média de idade: } \\
\mathrm{Gl}=14,2 \pm 2,2 \\
\mathrm{GC}=14,3 \pm 1,8\end{array}$ & $\begin{array}{l}45 \text { minutos } \\
3 \text { dias por semana } \\
12 \text { semanas }\end{array}$ & $\begin{array}{l}\text { Hemoglobina glicada } \\
\text { Dose diária de insulina } \\
\text { subcutânea } \\
\text { HDL-c } \\
\text { Triglicerídeos } \\
\text { Colesterol total } \\
\text { LDL-c } \\
\text { HDL-c }\end{array}$ & $\begin{array}{l}\text { Não foram verificadas diferenças } \\
\text { estatisticamente significativas na } \\
\text { hemoglobina glicada e nas doses } \\
\text { diárias de insulina subcutânea entre } \\
\text { os grupos }\end{array}$ \\
\hline $\begin{array}{l}\text { Fourie } M \text { et al. [28] } \\
2013 \\
\text { África do Sul }\end{array}$ & $\begin{array}{l}50 \text { idosas sedentárias foram } \\
\text { divididas em } 2 \text { grupos: } 25 \text { no Gl } \\
\text { e } 25 \text { no GC. } \\
\text { Média de idade: } \\
\text { GC }=65,32 \pm 5,01 \\
\text { Gl }=66,12 \pm 4,77\end{array}$ & $\begin{array}{l}60 \text { minutos } \\
3 \text { dias por semana } \\
8 \text { semanas }\end{array}$ & $\begin{array}{l}\text { Pressão arterial } \\
\text { Glicemia } \\
\text { HDL-c } \\
\text { Triglicerídeos } \\
\text { Colesterol total } \\
\text { IMC }\end{array}$ & $\begin{array}{l}\text { As idosas do Gl tiveram uma } \\
\text { diminuição estatisticamente } \\
\text { significativa da pressão arterial } \\
\text { sistólica }(p=0,040) \text { e aumento níveis } \\
\text { de glicose }(p<0,001)\end{array}$ \\
\hline $\begin{array}{l}\text { Ramezankhany A et al. [29] } \\
2012 \\
\text { Irã }\end{array}$ & $\begin{array}{l}46 \text { mulheres sedentárias } \\
\text { foram divididas em } 4 \text { grupos: } \\
\text { 1) Pilates } \\
\text { 2) Exercícios aeróbicos } \\
\text { 3) Dieta hipocalórica } \\
\text { 4) Controle (GC) } \\
\text { Média de idade: } \\
G C=36,4 \pm 2,99 \\
\text { Dieta }=36,8 \pm 2,91 \\
\text { Exercícios aeróbicos }=35,16 \pm 2,94 \\
\text { Pilates }=37,16 \pm 2,88\end{array}$ & $\begin{array}{l}45 \text { minutos } \\
3 \text { dias por semana } \\
16 \text { semanas }\end{array}$ & $\begin{array}{l}\text { Relação Cintura e quadril, } \\
\text { HDL-c } \\
\text { LDL-c } \\
\text { Triglicerídeos } \\
\text { Colesterol total } \\
\text { IMC }\end{array}$ & $\begin{array}{l}\text { Foram encontradas diferenças } \\
\text { estatisticamente significativas em } \\
\text { relação cintura-quadril (RCQ) dentro } \\
\text { de todos os grupos experimentais } \\
(\mathrm{P}=0,001) \text { e no IMC do grupo dos } \\
\text { exercícios aeróbicos e do Pilates } \\
(\mathrm{P}<0,05)\end{array}$ \\
\hline $\begin{array}{l}\text { Cakmakçi O. [30] } \\
2011 \\
\text { Turquia }\end{array}$ & $\begin{array}{l}58 \text { mulheres obesas e } \\
\text { sedentárias, } \\
34 \text { no } \mathrm{Gl} \text { e } 27 \text { no GC. } \\
\text { Média de idade: } \\
\mathrm{Gl}=36,15 \pm 9,59 \\
\mathrm{GC}=38,96 \pm 10,02\end{array}$ & $\begin{array}{l}60 \text { minutos } \\
4 \text { dias por semana } \\
8 \text { semanas }\end{array}$ & $\begin{array}{l}\text { IMC } \\
\text { Relação cintura/quadril } \\
\text { Circunferência da cintura } \\
\text { Porcentagem de gordura } \\
\text { corporal } \\
\text { Massa gorda e magra }\end{array}$ & $\begin{array}{l}\text { O GI apresentou diferença } \\
\text { estatisticamente significativa na } \\
\text { relação cintura quadril }(P<0,001) \text { e } \\
\text { nos percentuais de gordura corporal } \\
(P<0,001) \text { e massa gorda }(P<0,001)\end{array}$ \\
\hline $\begin{array}{l}\text { Cakmakçi O. [31] } \\
2012 \\
\text { Turquia }\end{array}$ & $\begin{array}{l}36 \text { mulheres com sobrepeso e } \\
\text { sedentárias foram randomizadas. } \\
20 \text { para grupo de intervenção e } \\
16 \text { grupo controle } \\
\text { Média de idade: } \\
\mathrm{Gl}=28,35 \pm 10,18 \\
\mathrm{GC}=28,19 \pm 7,44\end{array}$ & $\begin{array}{l}60 \text { minutos } \\
3 \text { dias por semana } \\
10 \text { semanas }\end{array}$ & $\begin{array}{l}\text { IMC } \\
\text { Relação cintura-quadril } \\
\text { Circunferência da cintura } \\
\text { Composição corporal, } \\
\text { Massa gorda e magra }\end{array}$ & $\begin{array}{l}\text { Foram encontradas diferenças } \\
\text { estatisticamente significativas em } \\
\text { massa gorda e magra }(p<0,001) \text {, } \\
\text { relação cintura quadril e IMC } \\
(p<0,001)\end{array}$ \\
\hline $\begin{array}{l}\text { Martins-Meneses DT et al. [32] } \\
2014 \\
\text { Brasil }\end{array}$ & $\begin{array}{l}44 \text { mulheres hipertensas tratadas } \\
\text { com medicação } \\
\mathrm{Gl}=51,8 \pm 4,3 \\
\mathrm{GC}=49,0 \pm 7,5\end{array}$ & $\begin{array}{l}60 \text { minutos } \\
2 \text { dias por semana } \\
16 \text { semanas }\end{array}$ & $\begin{array}{l}\text { Pressão arterial } \\
\text { Frequência cardíaca } \\
\text { IMC } \\
\text { Relação cintura quadril }\end{array}$ & $\begin{array}{l}\text { Foram encontradas diferenças } \\
\text { estatisticamente significativas nas } \\
\text { médias da pressão arterial diastólica e } \\
\text { sistólica, na circunferência da cintura } \\
\text { e na relação cintura quadril }(p<0,05)\end{array}$ \\
\hline
\end{tabular}

GI: grupo intervenção com Pilates; GC: grupo controle; IMC: índice de massa corporal; HDL-c: lipoproteína de alta densidade ligada ao colesterol;

LDL-c: lipoproteína de baixa densidade ligada ao colesterol. 
Dos sete estudos analisados, três $(42,86 \%)$ investigaram o efeito do Pilates em fatores de risco cardiometabólicos clássicos: triglicerídeos, glicose e lipoproteína de alta densidade ligada ao colesterol (HDL-c), sendo que um estudo verificou aumento significativo dos níveis de glicose, tanto no grupo Pilates $(\mathrm{P}<0,0001)$ quanto controle $(\mathrm{P}=0,001)$ e outro mostrou aumento dos níveis de HDL-c somente no grupo controle $(\mathrm{P}=0,046)$ [27-29]. Já o estudo de Ramezankhany et al., não demonstrou diferenças estatisticamente significativas entre os grupos com relação a esses fatores $(\mathrm{P}>0,05)$ [29]. Porém, desses três estudos, dois deles também avaliaram concomitantemente outros parâmetros, como pressão arterial sistêmica, IMC e relação cintura/quadril, verificando reduções estatisticamente significativas no grupo que realizou Pilates [28,29]. Dos sete estudos incluídos, seis deles $(85,71 \%)$ mostraram reduções estatisticamente significativas entre os grupos intervenção com Pilates e controle, em algum dos seguintes parâmetros: níveis de pressão arterial sistólica e diastólica [28,32] IMC [29], porcentagem de gordura corporal [26, 30], relação cintura/ quadril [29,31] e massa gorda [30, 31] (Tabela 1).

Do total de artigos analisados, a maioria encontrou efeitos positivos do Pilates na composição corporal, no IMC e na relação cintura/quadril, como pode ser observado nos estudos realizados por Cakmakçi [30, 31], Fourie et al. [26] e Ramezankhany et al. [29]. Esses estudos indicaram que o Pilates teve efeito benéfico sobre as variáveis antropométricas e composição corporal quando praticado durante 45 a 60 minutos por dia, de duas a quatro vezes por semana, em um período compreendido entre oito e 10 semanas. O Pilates, apesar de não ser considerado aeróbico por completo, produz um elevado gasto energético, com consequente alteração no metabolismo celular. Dessa forma, exerce efeitos positivos no desempenho físico e na composição corporal [30,31,33]. É importante ressaltar que exercícios físicos como o Pilates desencadeiam uma série de alterações fisiológicas, principalmente na regulação da glicemia e na deposição de gordura abdominal, refletindo-se no aumento da sensibilidade à insulina, que atua no controle glicêmico, síntese proteica e na regulação da estocagem de gordura $[34,35]$. Entretanto, a busca na literatura resultou em três estudos [27-29] que não observaram efeitos positivos do Pilates nos níveis de glicose, dos triglicerídeos e da HDL-c, que em níveis alterados e em conjunto caracterizam a síndrome metabólica. Um desses estudos [27] analisou o efeito do Pilates em um grupo de diabéticos, mas não encontrou diferença estatística entre as médias de hemoglobina glicada e nas doses diárias de insulina subcutânea, entre os grupos intervenção e controle. Uma das hipóteses aventadas pelos autores do estudo para tal resultado seria que os participantes aumentaram a ingestão de carboidratos antes da atividade, devido ao risco de hipoglicemia durante a realização do exercício. Contudo, a ingestão calórica dos participantes da pesquisa não foi avaliada [27].

As dislipidemias são outros fatores de risco para doenças cardiometabólicas, que associados ao sedentarismo aumentam ainda mais o risco cardiovascular [36]. Na presente revisão, os estudos que analisaram o perfil lipídico não encontraram diferenças relacionadas às médias de triglicerídeos, HDL-c, LDL-c e colesterol total [27-29] nos grupos que realizaram o Pilates. Umas das justificativas referidas pelos autores para tal resultado, é que apenas os exercícios aeróbicos podem exercer, de fato, alguma influência nas dislipidemias. Em uma metanálise realizada para verificar o impacto de ensaios clínicos randomizados que utilizaram exercícios físicos para a perda de peso em pessoas com sobrepeso/obesidade, demonstrou-se que a atividade aeróbica, mesmo quando não reduzindo o peso, promove diminuição dos triglicerídeos totais, pressão arterial e glicemia de jejum [37]. Entretanto, o método Pilates não pode ser considerado uma modalidade de exercício aeróbico, caracterizando-se como um exercício de força e resistência com capacidade de reduzir a adiposidade abdominal e visceral, que são reservas ricas em triglicerídeos [30]. Nesse sentido, o Pilates pode contribuir para o melhor metabolismo dos carboidratos e da oxidação das gorduras, reduzindo os níveis de lipídeos circulantes e intramusculares, melhorando a sensibilidade à insulina e prevenindo o desenvolvimento do diabete mellitus tipo 2 e da hipertensão. Outra possível explicação para o achado de ausência de efeito do Pilates no perfil lipídico seria o uso das estatistinas pelos indivíduos investigados. Isto porque estudos com outra modalidade de exercicio físico, mesmo com baixa intensidade, porém com maior frequência, demonstraram um impacto benéfico no perfil lipídico, inclusive de idosos [38].

Por outro lado, dois estudos incluídos nesta revisão encontraram efeitos benéficos do Pilates na pressão arterial sistêmica. Fourie et al. [28] avaliaram o efeito do treinamento do Pilates na pressão arterial de idosas e verificaram que o grupo de intervenção apresentou uma redução significativa nas médias da pressão arterial sistólica durante o treinamento. Martins-Meneses et al. [32] também encontraram resultados semelhantes nos níveis tanto da pressão 
sistólica quanto da diastólica em seu estudo. Esses resultados foram atribuídos pelos autores às técnicas de respiração, que podem estar associadas à diminuição da tensão, resultando no relaxamento do organismo, tendo como consequência redução na pressão arterial. Além disso, através da prática de exercício físico de resistência e força como o Pilates, ocorre um aumento da força de ejeção do sangue na parede vascular. Isso gera maior capacidade de produção de óxido nítrico no endotélio, proporcionando um efeito vasodilatador na circulação arterial. Adicionalmente, a atividade física reduz a atividade simpática, melhorando também o tônus vascular [39-41].

A hipertensão é um dos fatores de risco cardiovascular mais importantes, pois é responsável por, aproximadamente, $45 \%$ das doenças cardíacas, como infarto agudo do miocárdio e angina instável, e $51 \%$ dos acidentes vasculares cerebrais [42]. Portanto, o controle da pressão arterial é fundamental para a redução e controle das DCV e doenças cerebrovasculares. Nesse sentido, a prática de Pilates pode ser um fator coadjuvante no controle, manejo ou mesmo remissão da hipertensão e seus desfechos negativos.

As principais limitações deste estudo foram o reduzido número de publicações com a resposta à pergunta norteadora e a escassez de estudos com populações brasileiras. A população brasileira apresenta um perfil étnico, genético, socioeconômico e de saúde diverso e, provavelmente, muito diferente das populações investigadas nos estudos incluídos nesta revisão, o que pode prejudicar a generalização dos resultados obtidos. Outro fator limitante é que a maioria dos estudos investigou o Pilates para melhora da dor lombar crônica, correção postural, aumento de flexibilidade e alteração de composição corporal, resultando em um número reduzido de estudos incluídos nesta revisão. Além disso, apenas um estudo foi encontrado investigando o Pilates em diabéticos [27], e nenhum com amostras de hipertensos e dislipidêmicos, apesar de serem analisados perfil lipídico e pressão arterial dos sujeitos da pesquisa antes e após a intervenção. Adicionalmente, apenas um estudo, o qual não foi incluído nesta revisão [17], investigou o hábito alimentar, e nenhum estudo incluído avaliou o uso de medicamentos hipolipemiantes dos sujeitos da pesquisa, o que pode ter se configurado um fator interveniente na obtenção dos resultados relacionados à glicemia e ao perfil lipídico.

Também é importante ressaltar o tempo de duração da intervenção do Pilates nos estudos aqui incluídos. Apenas dois estudos realizaram o Pilates por 16 semanas, tendo encontrado efeitos benéficos na relação cintura/ quadril, circunferência abdominal, IMC e pressão arterial sistêmica [29,32]. Além disso, não há uniformidade quanto à duração do tempo de intervenção nos estudos que analisaram o perfil lipídico e a glicemia dos indivíduos que realizaram o Pilates. Dos três estudos que investigaram o perfil lipídico e a glicemia, um foi realizado por 12 semanas [27], outro por oito semanas [28] e o terceiro por 16 semanas [29], não apresentando efeitos benéficos nessas variáveis. O estudo realizado por Fourie et al. [28] verificou um aumento estatisticamente significativo nos níveis glicêmicos tanto no grupo controle quanto no Pilates $(\mathrm{P}<0,001)$. O que dificulta inferir sobre a real eficácia do método nos fatores de risco cardiometabólicos clássicos.

\section{CONSIDERAÇÕES FINAIS}

O Pilates está se consolidando como uma modalidade de exercício físico eficaz na redução de porcentagem de gordura corporal, da massa gorda, da relação cintura/ quadril e da pressão arterial em indivíduos de diferentes faixas etárias. Aproximadamente $86 \%$ dos estudos incluídos na presente revisão mostraram a eficácia do Pilates na redução de algum desses parâmetros corporais e fisiológicos. Esses benefícios, principalmente a redução da obesidade abdominal, relação cintura/ quadril e pressão arterial, têm um impacto clinicamente relevante na morbimortalidade por DCV e acidente vascular cerebral, bem como no aumento da capacidade funcional e na qualidade de vida. Entretanto, apenas três estudos foram encontrados investigando o efeito do Pilates em relação de fatores de risco clássicos para as doenças cardiometabólicas, como a glicemia, os triglicerídeos e a HDL-c, não sendo observados efeitos do Pilates nesses fatores. Considera-se, portanto, que são necessários mais estudos de intervenção com o Pilates (e não somente o com o Mat Pilates), com amostras bem definidas e talvez com duração de intervenção maior para que se possa confirmar a eficácia do método na remissão dos fatores de risco cardiometabólicos, com vistas à prevenção das doenças associadas.

\section{AGRADECIMENTO}

Ao Programa Nacional de Pós-Doutorado da Coordenação de Aperfeiçoamento de Pessoal de Nível Superior-PNDP/CAPES. 


\section{REFERÊNCIAS}

1. Brasil. Ministério da Saúde. Cadernos de Atenção Básica, no. 38, 2014. [cited 2015 March] Available at: http://bvsms.saude.gov.br/bvs/ publicacoes/estrategias_cuidado_doenca_cronica_obesidade_cab38.pdf

2. National Cholesterol Education Program - NCEP. Executive Summary of the Third Report of the National Cholesterol Education Program. Expert Panel on Detection, Evaluation, and Treatment of High Blood Cholesterol in Adults - ATPIII. JAMA. 2001;285:2486-2497. http:// dx.doi.org/10.1001/jama.285.19.2486

3. Diretrizes da Sociedade Brasileira de Cardiologia. Pocket Book.5 edição 2011-2013. p.4-446.

4. González-Gross M, Meléndez A. Sedentarism, active lifestyle and sport: Impact on health and obesity prevention. Nutr Hosp. 2013;28(Suppl 5):89-98. http://dx.doi.org/10.3305/nh.2013.28.sup5.6923

5. Nauman J, Nilsen TI, Wisloff U, Vatten LJ. Combined effect of resting heart rate and physical activity on ischaemic heart disease: mortality follow-up in a population study (the HUNT study, Norway). J Epidemiol Community Health. 2010;64:175-81. http://dx.doi.org/10.1136/ jech.2009.093088

6. Myers J, Prakash M, Froelicher V, Do D, Partington S, Atwood JE. Exercise capacity and mortality among men referred for exercise testing. N Engl J Med. 2002;346:793-801. http://dx.doi.org/10.1056/NEJMoa011858

7. Aravindalochanan V1, Kumpatla S, Rengarajan M, Rajan R, Viswanathan V. Risk of diabetes in subjects with sedentary profession and the synergistic effect of positive family history of diabetes. Diabetes Technol Ther. 2014;16(1):26-32. http://dx.doi.org/10.1089/dia.2013.0140

8. Brasil. Ministério da Saúde, Secretaria de Atenção à Saúde. Departamento de Atenção Básica. [Internet] Brasília: 2010 [cited 2015 Jan 18]. Available at: http://bvsms.saude.gov.br/bvs/publicacoes/caderno atencao primaria 29 rastreamento.pdf

9. Junges S, Gottlieb MG, Babtista RR, Quadros CB, Resende TL,Gomes I. Eficácia do método Pilates para a postura e flexibilidade em mulheres com hipercifose. Rev Bras Ciênc Mov. 2012;20(1):21-23.

10. Gonzalvo-Aladro AR, Diaz MM, Jimenez JM, Elizondo JH, Vargas GA. The effect of Pilates exercises on body composition: A systematic review. J Body Mov Ther. 2012;16(1):109-14. http://dx.doi.org/10.1016/j.jbmt.2011.06.001

11. Shaw BS, Shaw I, Brown GA. Resistance training and its effect on total, central and abdominal adiposity. SAJRSPER. 2009;31:97-108.

12. Leung FP, Yung LM, Laher I, Yao X, Chen ZY, Huang Y. Exercise, vascular wall and cardiovascular diseases: an update (Part 1). Sports Med. 2008;38(12):1009-24. http://dx.doi.org/10.2165/00007256-200838120-00005

13. Ceriello A, Motz E. Is oxidative stress the pathogenic mechanism underlying insulin resistance, diabetes, and cardiovascular disease? The common soil hypothesis revisited. Arterioscler Thromb Vasc Biol. 2004;24(5):816-23. http://dx.doi.org/10.1161/01 ATV.0000122852.22604.78

14. Seidell JC, Visscher TLS, Hoogeveen, RT. Overweight and obesity in the mortality rate data: current evidence and research issues. Med Sci Sports Exerc. 1999;31(11):597-601. http://dx.doi.org/10.1097/00005768-199911001-00018

15. Pilates JH, Miller WJ. Pilates Return to life through Contrology. New York: JJ Augustin; 1945. Apud: Friedman P, Eisen G. The Pilates Method Physical and Mental Conditioning. London: Viking Studio; 2005.

16. Latey P. Updating. The principles of the Pilates method - part 2. J Bodyw Mov Ther. 2002;6:94-101. http://dx.doi.org/10.1054/ jbmt.2002.0289

17. Natour J, Cazotti L de A, Ribeiro LH, Baptista AS, Jones A. Pilates improves pain, function and quality of life in patients with chronic low back pain: a randomized controlled trial. Clin Rehabil. 2015;29(1):59-68. http://dx.doi.org/10.1177/0269215514538981

18. Wells C, Kolt GS, Marshall P, Hill B, Bialocerkowski A. The effectiveness of Pilates exercise in people with chronic low back pain: a systematic review. PLoS One. 2014;9(7):e100402. http://dx.doi.org/10.1371/journal.pone.0100402

19. American College of Sports Medicine - ACSM. Mind/body exercise what is it? [Internet] 2008 [cited 2015 Jan 18] Available at: http:// www.acsm.org/2008-fall-fspn_mind-body-exercise

20. Egaña M, Smith S, Green S. Revisiting the effect of posture on high-intensity constant-load cycling performance in men and women. Eur J Appl Physiol. 2007;99(5):495-501. http://dx.doi.org/10.1007/s00421-006-0365-8

21. Liberatti A, Altman DG, Tetzlaff J, Mulrow C, Gotzsche PC, Ioannidis JP. The Prisma statement for reporting systematic reviews and meta-analyses of studies that evaluated health care interventions: explanation and elaboration. PloS Med. 2009;62(10):1-34. http://dx.doi org/10.1371/journal.pmed.1000100

22. Vasconcellos MHO, Silva RDS, Santos SMB, Merlo JRC, Conceição TMA. The Pilates ${ }^{\circledR}$ Method in the treatment of lower back pain Fisioter Mov. 2014;27(3):459-67. http://dx.doi.org/10.1590/0103-5150.027.003.AR01

23. Guimarães ACA, Azevedo SF, Simas JPN, Machado Z, Jonck VTF. The effect of Pilates method on elderly flexibility. Fisioter Mov. 2014;27(2):181-8. http://dx.doi.org/10.1590/0103-5150.027.002.AO03

24. Araújo MEA, Silva EB, Vieira PC, Cader AS, Mello DB, Dantas EHM. Redução da dor crônica associada à escoliose não estrutural, em universitárias submetidas ao método Pilates. Motriz. 2010;16(4):958-66. http://dx.doi.org/10.5016/1980-6574.2010v16n4p958

25. Costa LMR, Roth A, Noronha M. O método Pilates no Brasil: uma revisão de literatura. Arq Catarin Med. 2012;41(3):87-92.

26. Fourie M, Gildenhuys GM, Shaw I, Shaw BS, Toriola AL, Goon DT. Effects of a Mat Pilates programme on body composition in elderly women. West Indian Med J. 2013;62(6):524-8. http://dx.doi.org/10.7727/wimj.2012.107

27. Tunar M, Ozen S, Gaksen D, Asar G, Bedizds S, Darcan S. The effects of Pilates on metabolic control and physical performance in adolescentes with type 1 diabetes mellitus. J Diabetes Complications. 2012;26(4):348-51. http://dx.doi.org/10.1016/j.jdiacomp.2012.04.006

28. Fourie M, Gildenhuys M, Shaw I, Shaw B, Toriola A, Goon DT. Effects of a Mat Pilates program on cardiometabolic parameters in elderly women. Pak J Med Sci. 2013; 29(2):500-4. http://dx.doi.org/10.12669/pjms.292.3099 
29. Ramezankhany A, Ali NP, Hedayati M. Comparing effects of aerobics Pilates exercise and low calorie diet on leptina levels and lipid profiles in sedentary women. Iran J Basic Med Sci. 2011;14(3)257-61.

30. Cakmakçi O. The Effect of 8 Week Plates Exercise on Body Composition in Obese Women. Coll Antropol. 2011;35(40):1045-50.

31. Cakmakçi O. The effect of 10 week Pilates Mat exercise program on weight loss and body composition for overweight Turkish women. World Appl Sci J. 2012;19(3):431-8. http://dx.doi.org/105829/idosi.wasj.2012.19.03.631

32. Martins Meneses DT, Antunes HK, Oliveira NR, Medeiros A. Mat Pilates training reduced clinical and ambulatory blood pressure in hypertensive women using antihypertensive medications. Int J Cardiol. 2014;179:262-8. http://dx.doi.org/10.1016/j.ijcard.2014.11.064

33. Kloubec JA. Pilates for improvement of muscle endurance, flexibility, balance, and posture. J Strength Cond Res. 2010;24(3):661-7. http:// dx.doi.org/10.1519/JSC.0b013e3181c277a6

34. Chien KL. Chen MF, Hsu HC, Ta-Chen Su, Lee YT. Sports activity and risk of type 2 diabetes in Chinese. Diabetes Res Clin Pract. 2009;84(2):311-8. http://dx.doi.org/10.1016/j.diabres.2009.03.006

35. Zanuso S, Jimenez A, Pugliese G, Corigliano G, Balducci S. Exercise for the management of type 2 diabetes: a review of the evidence. Acta Diabetol. 2010;47:15-22. http://dx.doi.org/10.1007/s00592-009-0126-3

36. Kelley GA, Kelley KS. Aerobic exercise and lipids and lipoproteins in children and adolescents: a meta-analysis of randomized controlled trials. Atherosclerosis. 2007;191(2):447-53. http://dx.doi.org/10.1016/j.atherosclerosis.2006.04.019

37. Shaw K, Gennat H, O’Rourke P, Del Mar C. Exercise for overweight or obesity. Cochrane Database Syst Rev. 2006;(4):CD003817. http:/ dx.doi.org/10.1002/14651858.CD003817.pub3

38. Pescatello LS. Low intensity physical activity benefits blood lipids and lipoproteins in older adults living at home. Age Ageing. 2000;29(5):433-9. http://dx.doi.org/10.1093/ageing/29.5.433

39. Viecili PR, Bündchen DC, Richter CM, Dipp T, Lamberti DB, Pereira AM, Barbosa Lde C, Rubin AC, Barbosa EG, Panigas TF. Curva dose-resposta do exercício em hipertensos: análise do número de sessões para efeito hipotensor. Arq Bras Cardiol. 2009;92(5):361-7. http://dx.doi.org/10.1590/S0066-782X2009000500010

40. Bundchen DC. Schenkel IC. Exercício físico controla a pressão arterial e melhora a qualidade de vida. Rev Bras Med Esporte. 2013;19(2): 91-5. http://dx.doi.org/10.1590/S1517-86922013000200003

41. Silva AS, Zanesco A. Exercício físico, receptores beta adrenérgicos e resposta vascular. J Vasc Bras. 2010;9(2):48-56. http://dx.doi org/10.1590/S1677-54492010000200007

42. World Health Organization (WHO). World Health Organization 2008-2013 Action Plan for the Global Strategy for the Prevention and Control of Noncommunicable Diseases. Noncommunicable diseases country profiles 2011. [Internet ] Washington (DC): 2011 [cited 2015 Jan 18] Available at http://www.who.int/nmh/publications/ncd_action_plan_en.pdf 